\title{
Habilidades Sociais e Características Pessoais em Escolares de Belém
}

\author{
Social Skills and Personal Features at Belém Students
}

\author{
Thaciana Araujo da Silva* \& Lilia Iêda Chaves Cavalcante \\ Universidade Federal do Pará, Belém, PA, Brasil
}

\begin{abstract}
Resumo
Este estudo relacionou a frequência da emissão de reação socialmente habilidosa, não-habilidosa passiva e não-habilidosa ativa às variáveis da criança (sexo, idade, condições clínicas). Participaram 57 meninos e 52 meninas, entre seis e 12 anos. O Questionário de Caracterização da Criança (QCC) levantou informações sobre sexo, idade e condições clínicas e o Inventário Multimídia de Habilidades Sociais de Crianças (IMHSC-Del Prette) avaliou as habilidades sociais, respondidos pelos cuidadores e escolares, respectivamente. Utilizou-se os testes $U$ de Mann-Whitney, o coeficiente de correlação de Pearson e o teste $t$ para análises dos dados. Os resultados indicaram: (a) diferenças significativas na adequação das reações habilidosas e não-habilidosas $(p<0,001)$; (b) aumento do repertório de habilidades sociais conforme o avançar dos anos $(p<0,001)$; e (c) meninas apresentaram mais frequência de habilidades sociais que meninos $(p=0,040)$. Não houve associações significativas entre habilidades sociais e condições clínicas $(p=0,539)$. Verificou-se que sexo e idade podem interferir na presença e desempenho de repertório socialmente habilidoso. Sugerem-se outras técnicas de avaliação que complementem os dados investigados e possibilitem intervenções futuras para amostras semelhantes. Palavras-chave: Habilidades sociais, características pessoais, problemas de saúde.
\end{abstract}

\begin{abstract}
This study linked the emission frequency of socially skilled reaction, unskilled passive reaction and unskilled active reaction to the child's variables (gender, age, medical conditions). Participated 57 boys and 52 girls between six and 12 years old. The Child Characterization Questionnaire (QCC) collected data on sex, age and clinical conditions and the Social Skills of Children Multimedia Inventory (IMHSC-Del Prette) evaluated social skills answered by caregivers and students, respectively. It is used the Mann-Whitney $U$ test, Pearson's correlation coefficient and the $t$ test were used for data analysis. The results indicated: (a) significant differences in the adequacy of skilled and unskilled reactions $(p<.001)$; (b) increase in social skills repertoire as the years advance $(p<$ $.001)$; (c) higher frequency of social skills in girls than in boys $(p=.040)$. There were no significant associations between social skills and clinical conditions $(p=.539)$. The study observed that gender and age may interfere with the presence and performance of socially skilled repertoire. Other evaluation techniques may be suggested to complement the investigated data and allow for future interventions to similar samples.

Keywords: Social skills, personal characteristics, health issues.
\end{abstract}

A infância é marcada por inúmeras experiências que dependendo do seu percurso desenvolvimental (Bronfenbrenner, 2011) favorecem ou prejudicam a aquisição de habilidades sociais, e o seu déficit pode estar relacionado à presença de problemas de comportamento apresentados pelas crianças (Arslan, Durmusoglu-Saltali, \& Yilmaz, 2011; Moura, Marinho-Casanova, Meurer, \& Campana, 2008; Pavarino, Del Prette, \& Del Prette, 2005; Welsh, Parke, Widaman, \& O'Neil, 2001). Há evidências de que a expressão das habilidades sociais pode variar conforme diversos fatores, como sexo, idade e condições clínicas de saúde das crianças.

As habilidades sociais compõem "uma classe de comportamentos que um indivíduo emite para completar com sucesso uma tarefa social" (Del Prette \& Del Prette, 2009, p. 19). Dizem respeito à presença de um repertório comportamental que permite ao indivíduo ampliar a sua capacidade para resolução de problemas imediatos e minimizar outros por meio da construção de relacionamentos saudáveis e produtivos com as demais pessoas (Caballo, 2012), e possui dimensão comportamental, pessoal, situacional e cultural que influenciam a sua aprendizagem e manutenção (Caballo, 2012; Del Prette \& Del Prette, 2009). 
Segundo Del Prette e Del Prette (2005), existem três tipos de reações possíveis diante de uma situação de interação social, as reações não-habilidosas passivas, as não-habilidosas ativas e as habilidosas. As reações não-habilidosas passivas e as do tipo ativas são aquelas que prejudicam a competência social, sendo que o primeiro grupo reúne as que se expressam de forma encoberta, tais como incômodo, mágoa ou no sentido do não enfrentamento das demandas, enquanto que o segundo grupo é constituído por comportamentos que se apresentam de maneira aberta através de agressividade física ou verbal, ironia, negativismo, coerção e autoritarismo, comprometendo a competência social. As reações habilidosas, por sua vez, estariam em uma posição intermediária entre as já citadas, e são as que se expressam pela coerência entre os comportamentos abertos e encobertos, no sentido de promover a adequação às demandas e que contribuem para a competência social.

Em virtude da interferência que um repertório de habilidades sociais possui na vida da criança, admitem-se neste estudo que algumas variáveis que representam atributos pessoais podem direcionar certos padrões de comportamento socialmente desejáveis, tais como as classes de habilidades sociais de assertividade, civilidade e empatia. Entre as características pessoais que têm sido mais associadas à presença de habilidades sociais adequadas estão variáveis presentes no perfil pessoal da criança como sexo (Abdi, 2010; Cecconello \& Koller, 2000; Fumo, 2009; Garaigordobil \& Maganto, 2011; Valle \& Garnica, 2009), idade (Berry \& O'Connor, 2010; Bolsoni-Silva, Marturano, \& Freiria, 2010; Garaigordobil \& Maganto, 2011; Pichardo, García, Justicia, \& Llanos, 2008; Vugt, Dekovic, Prinzie, Stams, \& Asscher, 2013; Welsh et al., 2001) e condições de saúde (Carrillo et al., 2002; Ortiz et al., 2005; Salomão, Miyazaki, Cordeiro, Domingos, \& Valerio, 2008).

A relação entre comportamentos sociais habilidosos e não-habilidosos em crianças e a variável sexo, conforme a literatura investigada (Abdi, 2010; Cecconello \& Koller, 2000; Fumo, 2009; Garaigordobil \& Maganto, 2011; Valle \& Garnica, 2009), tem se expressado em maiores índices de comportamentos habilidosos (empatia, por exemplo) em meninas, em comparação aos meninos. O estudo de Abdi (2010), por exemplo, encontrou nos seus resultados que as meninas apresentaram escores mais altos de habilidades sociais (assertividade e cooperação) que meninos, e estes apresentam mais problemas de comportamentos externalizantes e hiperatividade que as meninas, mas sem diferenças entre os sexos para comportamentos internalizantes. Valle e Garnica (2009), também encontraram resultados semelhantes ao comparar as habilidades sociais em grupos de alunos de duas escolas e encontrou que na escola 1 , na avaliação das professoras, maiores médias de habilidades sociais estiveram associadas às crianças do sexo feminino, enquanto na escola 2 , as meninas destacaram-se com mais comportamentos habilidosos que os meninos tanto na autoavaliação quanto na das professoras.
Considera-se que as diferenças entre os sexos na emissão e déficits de classes de habilidades sociais podem ser explicados por fatores biológicos, como por exemplo, taxas hormonais de testoterona mais presente na emissão de comportamentos agressivos em meninos (Zahn-Waxler, Shirtcliff, \& Marceau, 2008), e culturais, através das formas de socialização diferenciadas para meninos e meninas, pois atuam diretamente na maneira como os padrões relacionais se desenvolvem, por exemplo, por meio de modelos aprendidos que reforçam os papeis de gênero masculino e feminino e que estão presentes nas relações das crianças com seus pares (Abdi, 2010; Caballo, 2012; Cecconello \& Koller, 2000).

Outro fator apontado pela literatura que está associado às variações no repertório social de crianças está relacionado à variável idade, evidenciando que gradualmente as crianças tendem a aprimorar seu repertório de habilidades sociais conforme vão crescendo. Berry e O'Connor (2010) ao avaliarem as habilidades sociais de crianças da pré-escola ao sexto ano escolar identificaram uma trajetória curvilínea das habilidades sociais, com períodos de aceleração/crescimento nos períodos da pré-escola ao primeiro ano, e do terceiro ao quinto ano. O estudo de Vugt et al. (2013) acompanharam crianças de 7 a 13 anos, após um ano da participação em programa de treino de habilidades sociais, e identificaram aumento no repertório social e menos problemas de comportamento nos dois grupos, demostrando que apesar de no grupo experimental a melhora ser mais evidente, as crianças do grupo controle também tiveram ganhos ao longo de um ano. Por sua vez, Bolsoni-Silva et al. (2010), ao compararem crianças com e sem indicativos de problemas de comportamentos em dois momentos (5 e 10 anos), observaram alterações significativas em seus repertórios sociais com o avanço da idade dos participantes, através do aumento da incidência de comportamento socialmente habilidoso no grupo de problemas de comportamento e diminuição no grupo designado socialmente habilidoso. Esses e outros estudos (Berry \& O'Connor, 2010; Boyer \& Nelson, 2015; Pichardo et al., 2008; Sabol \& Pianta, 2011; Welsh et al., 2001) indicam que essas alterações captadas pelo aumento do repertório social longitudinalmente é algo esperado em termos desenvolvimentais quando considerada a idade cronológica da criança. As crianças passam a se ver de forma mais independentes conforme a idade avança e recorrem menos a ajuda de adultos para resolução de conflitos, bem como tornam-se mais amadurecidas emocionalmente com o passar do tempo (Bee, 2003; Cecconello \& Koller, 2000; Pavarino et al., 2005).

Outro aspecto associado a variáveis que aumentam a probabilidade de o indivíduo apresentar em seu repertório de comportamentos sociais inadequados tem sido o estado de saúde. Alguns estudos apontam interferência do estado clínico de saúde da criança em seu ajustamento social quando esta apresenta doenças como asma, dermatoses e epilepsia (Carrillo et al., 2002; Lima, Guerra, \& Lemos, 2010; Zbikowski \& Cohen, 1998). As crianças podem 
apresentar sentimentos de inadequação no seu autoconceito apresentando quadros de problemas de comportamentos internalizantes, como ansiedade e depressão (Gon, Menezes, Jacovozzi, \& Zazula, 2013; Ortiz et al., 2005), além disso, patologias podem afetar diretamente a execução de atividades das crianças, como as lúdicas e de esporte, que muitas vezes são realizadas com pares, e as crianças são restringidas a participarem (Carrillo et al., 2002; Ortiz et al., 2005), afetando inclusive a sua qualidade de vida (Campanha, Freire, \& Fontes, 2008; Pílger \& Abreu, 2007; Silva, Naspitz, \& Solé, 2000). O estudo de Salomão et al. (2008), indicou frequente associação entre a presença de doença crônica como asma, por exemplo, em crianças e prejuízos nos níveis de competência social global, além da manifestação de transtornos comportamentais do tipo internalizante (ansiedade e depressão). Na mesma direção, Ortiz et al. (2005) encontraram em crianças com asma 98\% de ansiedade, além de interferências na participação em atividades lúdicas e esportes. Carrillo et al. (2002), ao investigar qualidade de vida em crianças com epilepsia também identificou comprometimento em atividades sociais em função do próprio estigma da doença e de restrições da doença que leva ao isolamento do enfermo da convivência de amigos e familiares, afetando o processo de socialização da criança.

Entende-se, assim, que déficits em habilidades sociais podem se manifestar em razão da presença de condições clínicas específicas (ser portador de doenças crônicas como a asma, ou depressão, por exemplo). Em outras palavras, os déficits em habilidades sociais podem ser explicados por eventos múltiplos, ora decorrentes de disposições biológicas, ora da interação entre o organismo com o ambiente, o que significa dizer que dependendo das particularidades da doença e da maneira como é enfrentada pelo paciente, várias áreas da sua condição psicossocial tendem a ser afetadas, incluindo, no caso de crianças, o relacionamento social e a vida escolar (Carrillo et al., 2002; Pílger \& Abreu, 2007; Silva, 2001; Silva et al., 2000).

Pelo exposto, admite-se que características pessoais como sexo, idade e condições clínicas são importantes por se considerar que podem atuar nos processos em interação com o ambiente ao longo da trajetória de desenvolvimento do indivíduo (Bronfenbrenner, 2011), podendo afetar os padrões de comportamento social conforme o estágio que a criança se encontra. Ou seja, a interação social poderá variar conforme estas variáveis, se meninos e com menos idade, pode haver mais conflitos com pares, por exemplo. E em virtude de sua avaliação na infância auxiliar na identificação de déficits de habilidades sociais e na geração de estratégias de promoção de repertório social adequados em escolares, com fins de prevenção de problemas de relacionamento e emocionais, existe um interesse em conhecer na região de Belém as habilidades sociais de escolares relacionados a estas variáveis, visto que ainda é um tema pouco explorado no Norte do país, de acordo com algumas revisões de literatura sobre o assunto (Bolsoni-Silva et al., 2006; Fumo, Manolio, Bello, \& Hayashi, 2009).
A partir de uma breve revisão da literatura sobre as possíveis associações entre habilidades sociais e variáveis pessoais, e da importância de se verificar como esta relação se apresenta em população de escolares da região Norte do país e em um único estudo, o objetivo do presente estudo pode ser assim descrito: relacionar a Frequência da emissão de reação socialmente habilidosa, não-habilidosa passiva e não-habilidosa ativa às variáveis da criança (sexo, idade, condições clínicas e tratamento de saúde) e avaliar os escores de habilidades sociais geral das crianças conforme seus indicadores de Frequência, Adequação e Dificuldade.

\section{Método}

\section{Participantes}

Este estudo é descritivo exploratório e de abordagem correlacional. Participaram deste estudo 109 crianças com idades entre 6 e 12 anos $(M=8,75 ; D P=1,35)$, que cursavam do $2^{\circ}$ ao $5^{\circ}$ ano do ensino fundamental de uma escola pública pertencente a uma instituição filantrópica que atende crianças da periferia da cidade de Belém. Também tomaram parte do estudo 100 cuidadores das crianças participantes, já que nove destes eram cuidadores de mais de uma criança que compunham a amostra. Os cuidadores que concordaram em colaborar, assim como autorizaram a participação dos filhos na pesquisa, assinaram o Termo de Consentimento Livre e Esclarecido (TCLE). Os critérios de exclusão abrangeram crianças cujos responsáveis não permitiram sua participação na pesquisa, e que apresentassem mais de 12 anos de idade. A seleção das crianças participantes foi sorteio a partir da lista dos nomes dos alunos por série e turma disponibilizados pela coordenação técnica pedagógica da escola. Foram selecionados 109 crianças de um total de 218 sendo $2^{\circ}$ ano $(n=23), 3^{\circ}$ ano $(n=30), 4^{\circ}$ ano $(n=27)$ e $5^{\circ}$ ano $(n=29)$. A seleção foi aleatória sem controle de variáveis como sexo, por exemplo.

\section{Instrumentos}

Para a avaliação do perfil biosociodemográfico dos participantes foi utilizado o Questionário de Caracterização da Criança (QCC), elaborado para esta pesquisa, composto por perguntas sobre I- Dados pessoais (10 itens); II- Composição e estrutura familiar; III-Escola; IV- Interesses e Rotina (10 perguntas abertas). Para este artigo foram selecionadas apenas as informações sobre idade, sexo e se a criança possuía algum problema de saúde, tais como dislexia, albinismo, asma, alergia, problemas na fala, epilepsia, rinite, otite, e se estava sendo submetida a algum tratamento de saúde.

Para avaliar as habilidades sociais das crianças o instrumento utilizado foi o Inventário Multimídia de Avaliação de Habilidades Sociais de Crianças- versão impressa (IMHSC-Del Prette; Del Prette \& Del Prette, 2005). O IMHSC-Del Prette foi elaborado por Del Prette e Del Prette (2005) para a autoavaliação da emissão de habilidades sociais por parte das crianças que cursam do $2^{\circ}$ ao $5^{\circ}$ ano quanto a aspectos de frequência (nunca $=0$, às 
Silva, T. A. \& Cavalcante, L. I. C. (2015). Habilidades Sociais e Características Pessoais em Escolares de Belém.

vezes $=1$, sempre $=2$ ), dificuldade (nenhuma $=0$, pouca $=1$, muita $=2$ ), adequação (errado $=0$, mais ou menos $=1$, certo $=2)$ em situações que representam o cotidiano da criança no contexto escolar e em outras situações de relacionamento com outras crianças e adultos. É composto por um caderno de pranchas que apresenta 21 situações e três formas de reação (não-habilidosa passiva, habilidosa e não-habilidosa ativa) para cada situação na forma de imagens digitalizadas. Os itens estão agrupados em quatro subescalas: Empatia e civilidade (itens 06, 10, 13, 14, 16, 18, 19, 21); Assertividade de enfrentamento (itens 03, 05, 11, 17, 20); Autocontrole (itens 02, 07, 09, 15); e Participação (itens 01, 08, 13); e mais dois itens que não se enquadram nessas subescalas, sendo agrupados e denominados de "Não-Fatores" $(04,12)$. Este instrumento apresenta consistência interna satisfatória para seus indicadores e validade para comparar o resultado de qualquer criança com os da amostra de referência através dos intervalos médios do escore total e escores de cada fator. Os indicadores de confiabilidade e validade foram aferidos através do Alpha de Conbach de 0,64 na consistência interna de Frequência e de 0,69 para Dificuldade. Informa-se ainda a presença de correlação positiva e significativa $(p<0,01)$ entre todos os itens do inventário e seus escores nos indicadores, conforme disposto no manual do instrumento.

\section{Procedimento}

Foi feito um contato com a instituição a fim de apresentar o projeto e solicitar autorização para execução da pesquisa. Em seguida a pesquisadora frequentou a escola para ambientar-se à sua rotina e atividades das crianças e para apresentação do projeto às professoras das séries pretendidas. A escolha dos participantes ocorreu por meio de sorteio, e em seguida procurou-se identificar quem eram as crianças e seus cuidadores. Foi enviada uma carta explicativa ao cuidador de cada criança para que este pudesse conhecer os objetivos e as condições em que se daria a participação dela e a sua própria. Foi explicada a necessidade de todos os responsáveis pela criança lerem e assinarem o TCLE. Após o recebimento do TCLE foi iniciada a coleta de dados com a aplicação individual do QCC com os cuidadores, na forma de entrevista com exceção de 12 deles que não puderam comparecer ou que não foi possível contatá-los. Nestes casos, o questionário foi preenchido em casa por eles e devolvido à pesquisadora. Em seguida, houve a aplicação do IMHSC-Del Prette com duplas de crianças em duas sessões (itens 1 a 11-primeira sessão; e segunda sessão dos itens 12 a 21). Cada sessão durou entre 30 a 40 minutos. A pesquisadora conduziu a aplicação do inventário que foi respondido pelas próprias crianças.

Para as análises dos dados, foram obtidos os valores médios correspondentes do escore total para cada indicador e tipo de reação e comparados aos valores médios de referência das reações habilidosas e não-habilidosas contidos no manual, através do teste $t$ de Student. Para as análises de diferenças intra-grupo, entre as médias de Frequência socialmente habilidosa e não-habilidosa passiva e ativa em relação às variáveis sexo, idade, condições clínicas, e tratamento de saúde, foi utilizado o teste não-paramétrico Mann-Whitney, visto que a distribuição da amostra não apresentou distribuição normal. Para a avaliação da correspondência linear entre a variável idade e os três tipos de reações (habilidosas, não-habilidosas passivas e não-habilidosas ativas) foi utilizado o coeficiente de correlação de Pearson.

\section{Cuidados Éticos}

A pesquisa foi realizada após a submissão e aprovação do projeto pelo Comitê de Ética, por atender às exigências previstas na Resolução no 466 de 12/12/2012. Os riscos que esta pesquisa ofereceu foram mínimos, pois não houve manipulação de qualquer material biológico ou execução de atividade que pudesse ameaçar a integridade física ou moral dos participantes. Além disso, os horários de aplicação dos instrumentos foram combinados previamente com as professoras das crianças participantes, garantindo que estas não seriam prejudicadas em seus estudos e/ou dinâmica escolar.

\section{Resultados}

Este estudo teve como objetivo relacionar a frequência da emissão de reação socialmente habilidosa, não-habilidosa passiva e não-habilidosa ativa às variáveis da criança (sexo, idade, condições clínicas e tratamento de saúde) e avaliar os escores de habilidades sociais gerais das crianças, segundo seus indicadores de Frequência, Adequação e Dificuldade.

A Tabela 1 apresenta a distribuição das frequências para categorias das variáveis biosociodemográficas e as médias das reações habilidosas, não-habilidosas passiva e ativa, conforme cada variável.

Os dados apresentados na Tabela 1 indicam que houve diferenças estatísticas entre as médias dos escores de Frequência de reações habilidosas para o sexo feminino em relação ao masculino, segundo o indicado pelo teste $U$ de Mann-Whitney. Ou seja, o sexo feminino apresentou maiores médias de reações habilidosas $(p=0,040)$ que o masculino, tendo sido observado o mesmo para a idade, com destaque para crianças na faixa etária de 9 a 12 anos ( $p$ $<0,001)$. Observa-se assim que, nesta amostra, as meninas a partir de nove anos de idade possuem um desempenho mais habilidoso socialmente que os meninos e que crianças abaixo desta faixa-etária.

As medidas de média e desvio-padrão estão dispostos na Tabela 2. Foi realizado o teste de correlação de Pearson para verificar a existência de associação entre idade e as reações habilidosas e não-habilidosas. O teste não indicou correlações entre idade e reações não-habilidosas passivas e não-habilidosa ativas $(r=0,019 ; p=0,843)$, 
Tabela 1

Comparação das Características Biosociodemográficas, Condições Clínicas e Tratamento de Saúde das Crianças conforme Frequência, Média e o p-Valor da Média do Indicador de Frequência de Reações Habilidosas e NãoHabilidosas a partir do IMHSC-Del Prette, conforme o Teste U de Mann-Whitney

\begin{tabular}{|c|c|c|c|c|c|c|}
\hline & Variáveis & Categorias & $\begin{array}{l}\text { Frequência } \\
\text { (\%) }\end{array}$ & HAB & $\begin{array}{l}\text { NHAB } \\
\text { Passiva }\end{array}$ & $\begin{array}{l}\text { NHAB } \\
\text { Ativa }\end{array}$ \\
\hline \multirow{6}{*}{$\begin{array}{l}\text { Características } \\
\text { biosociodemográficas }\end{array}$} & \multirow{2}{*}{ Sexo } & Masculino & $57(52,3)$ & 1,37 & 0,61 & 0,18 \\
\hline & & Feminino & $52(47,7)$ & 1,48 & 0,64 & 0,16 \\
\hline & \multirow{4}{*}{ Idade } & $p$-valor & & $0,040^{*}$ & 0,413 & 0,689 \\
\hline & & $6-8$ & $50(45,8)$ & 1,32 & 0,64 & 0,18 \\
\hline & & $9-12$ & $59(54,1)$ & 1,53 & 0,61 & 0,18 \\
\hline & & $p$-valor & & $p<0,001^{*}$ & 0,489 & 0,975 \\
\hline \multirow{6}{*}{$\begin{array}{l}\text { Condições } \\
\text { clínicas }\end{array}$} & \multirow{3}{*}{$\begin{array}{l}\text { Presença de condições } \\
\text { clínicas especíicas }\end{array}$} & Não & $89(81,7)$ & 1,42 & 0,62 & 0,17 \\
\hline & & Sim & $20(18,3)$ & 1,45 & 0,59 & 0,18 \\
\hline & & $p$-valor & & 0,539 & 0,730 & 0,778 \\
\hline & \multirow{3}{*}{ Tratamento de saúde } & Não & $97(89)$ & 1,42 & 0,61 & 0,16 \\
\hline & & Sim & $12(11)$ & 1,50 & 0,63 & 0,23 \\
\hline & & $p$-valor & & 0,314 & 0,816 & 0,135 \\
\hline
\end{tabular}

${ }^{*} p \leq 0,05$.

Tabela 2

Média e Desvio Padrão dos Tipos de Reações Habilidosas e Não-Habilidosas conforme Idade das Crianças

\begin{tabular}{ccccccccccc}
\hline & & \multicolumn{3}{c}{ Reação NH Passiva } & \multicolumn{2}{c}{ Reação NH Ativa } & \multicolumn{2}{c}{ Reação Habilidosa } \\
\cline { 3 - 10 } Idade & $n$ & Média & $D P$ & Mediana & Média & $D P$ & Mediana & Média & $D P$ & Mediana \\
\hline 6 & 4 & 0,60 & 0,34 & 0,53 & 0,12 & 0,15 & 0,08 & 1,08 & 0,33 & 1,00 \\
7 & 16 & 0,60 & 0,31 & 0,64 & 0,20 & 0,20 & 0,12 & 1,17 & 0,22 & 1,17 \\
8 & 30 & 0,65 & 0,27 & 0,69 & 0,16 & 0,19 & 0,12 & 1,42 & 0,23 & 1,43 \\
9 & 24 & 0,55 & 0,20 & 0,52 & 0,17 & 0,17 & 0,14 & 1,52 & 0,25 & 1,52 \\
10 & 25 & 0,69 & 0,23 & 0,71 & 0,18 & 0,20 & 0,10 & 1,57 & 0,24 & 1,57 \\
11 & 8 & 0,61 & 0,15 & 0,62 & 0,14 & 0,17 & 0,08 & 1,53 & 0,21 & 1,57 \\
12 & 2 & 0,38 & 0,07 & 0,38 & 0,31 & 0,30 & 0,31 & 1,26 & 0,78 & 1,26 \\
\hline
\end{tabular}

porém foi encontrada correlação positiva e significativa, ainda que fraca $(r=0,406 ; p<0,001)$, entre reações habilidosas e idade indicando que as habilidades sociais das crianças aumentaram conforme a idade. Supõe-se que o repertório de habilidades sociais tornou-se mais amplo à medida que a idade das crianças aumenta.

Os resultados gerais do teste $t$ sobre as médias de habilidades sociais da amostra conforme os tipos de reação e indicadores estão dispostos na Tabela 3. 
Silva, T. A. \& Cavalcante, L. I. C. (2015). Habilidades Sociais e Características Pessoais em Escolares de Belém.

Tabela 3

Média e Desvio Padrão dos Indicadores e Tipos de Reação do IMHSC-Del Prette comparadas às Medidas da Amostra em Estudo pelo Teste $t$

\begin{tabular}{ccccc}
\hline Reações & Indicadores & $\begin{array}{c}\text { Média de Referência } \\
\text { (Desvio Padrão) }\end{array}$ & $\begin{array}{c}\text { Média da amostra } \\
\text { (Desvio Padrão) }\end{array}$ & $p$-valor \\
\hline \multirow{2}{*}{$\begin{array}{c}\text { Socialmente Habili- } \\
\text { dosa }\end{array}$} & $\begin{array}{c}\text { Dificuldade } \\
\text { Frequência }\end{array}$ & $0,71(0,28)$ & $0,40(0,68)$ & $p<0,001^{*}$ \\
& Adequação & $1,44(0,23)$ & $1,43(0,69)$ & 0,880 \\
Não-habilidosa Pas- & $1,03(0,66)$ & $1,71(0,61)$ & $0,001^{*}$ \\
siva & Frequência & $0,68(0,26)$ & $0,62(0,73)$ & 0,391 \\
& Adequação & $0,37(0,35)$ & $0,80(0,83)$ & $p<0,001^{*}$ \\
Não-habilidosa Ativa & Frequência & $0,56(0,21)$ & $0,17(0,47)$ & $p<0,001^{*}$ \\
& Adequação & $0,65(0,48)$ & $0,18(0,52)$ & $p<0,001^{*}$ \\
\hline
\end{tabular}

$* p \leq 0,05$.

Sobre resultados do IMHSC-Del Prette, verifica-se, na Tabela 3, que comparada às médias de referência, houve diferenças significativas nos indicadores de Dificuldade nas reações habilidosas $(p<0,001)$, nos indicadores de Adequação nas reações habilidosas $(p<0,001)$ e não-habilidosas passivas $(p<0,001)$ e ativas $(p<0,001)$, e no indicador de Frequência nas reações não-habilidosas ativas $(p<0,001)$. Isto indica que as crianças da amostra apresentam menos frequentemente reações não-habilidosas ativas que as da amostra de referência do instrumento. Do mesmo modo, as crianças apresentaram mais facilidade em emitir reação habilidosa e consideram mais adequado reagir de forma habilidosa e não-habilidosa passiva, e menos adequado reagir de maneira não-habilidosa ativa, quando comparadas às médias das crianças da amostra de referência.

\section{Discussão}

Este artigo se propôs avaliar as habilidades sociais de crianças e verificar possíveis relações com características pessoais, tais como idade, sexo, condições clínicas e tratamento de saúde. Destaca-se que foram encontradas diferenças significativas em relação ao sexo, para reações habilidosas, o que confirma os achados de Abdi, (2010), Cecconello e Koller (2000), Fumo (2009), Garaigordobil e Maganto (2011) e Valle e Garnica (2009) que indicaram que meninas apresentaram um repertório comportamental social mais habilidoso que os meninos. Estes dados apontam para a necessária observação de variáveis de caráter situacional e cultural, uma vez que oferecem elementos importantes para se compreender determinados padrões de habilidades sociais encontrados de forma mais frequente entre meninos ou meninas (Abdi, 2010; Caballo, 2012; Cecconello \& Koller, 2000).

É possível que diferenças do repertório social entre meninas e meninos possam ser melhores explicadas quando apoiadas na forma diferenciada entre crianças do sexo masculino e feminino lidarem com as demandas do ambiente, e que são muitas vezes reforçadas por pessoas com as quais convivem. A natureza cultural das diferenças de papeis de gênero feminino e masculino gera a expectativa de os meninos apresentarem mais reações não-habilidosas que meninas (Caballo, 2012), já que estas tendem a se comportar de maneira mais habilidosa nas relações sociais. Elas tomam como referência os modelos e valores assimilados ao longo do seu processo de socialização (Abdi, 2010; Caballo, 2012; Cecconello \& Koller, 2000). A dimensão pessoal das habilidades sociais também deve ser considerada na avaliação de sua emissão conforme características cognitivas e de manejo do ambiente (Del Prette \& Del Prette, 2009), que evidenciam um repertório mais ou menos habilidoso em determinada situação.

Os resultados também confirmaram dados da literatura que defendem o pressuposto de que com o avançar da idade das crianças ocorre um aumento no repertório de habilidades sociais. No caso da amostra pesquisada, a partir de nove anos de idade as crianças apresentaram aumento em seu repertório de comportamentos socialmente habilidosos. Essa tendência tem sido explicada em razão do esperado crescimento e amadurecimento da criança com o passar do tempo (Berry \& O'Connor, 2010; Bolsoni-Silva et al., 2010; Boyer \& Nelson, 2015; Garaigordobil \& Maganto, 2011; Pichardo et al., 2008; Sabol \& Pianta, 2011; Vugt et al., 2013; Welsh et al., 
2001). Com o aumento da idade, crescem as experiências vividas pela criança em razão da sua inserção em novos contextos e grupos de convívio, o que traz demandas variadas de atuação no meio e uma maior diversificação das relações interpessoais. A consequência disso seriam inéditas oportunidades de aprendizagem e desenvolvimento do repertório de habilidades sociais (Bee, 2003; Berry \& O'Connor, 2010; Bolsoni-Silva et al., 2010; Cecconello $\&$ Koller, 2000; Pavarino et al., 2005).

No que se referem às variáveis clínicas específicas e de tratamento de saúde não foram encontradas diferenças significativas em relação à média de reações habilidosas e não-habilidosas. A literatura aponta que algumas doenças às vezes limitam a execução de tarefas escolares e atividades esportivas, interferindo, por exemplo, no desempenho escolar e na socialização (Carrillo et al., 2002; Lima et al., 2010; Ortiz et al., 2005; Zbikowski \& Cohen, 1998), além de prejuízos socioemocionais relativos à problemas internalizantes (Gon et al., 2013; Ortiz et al., 2005; Salomão et al., 2008; Silva, 2001), e na qualidade de vida da criança (Campanha et al., 2008). Pode-se pensar que a presença de condições clínicas apresentadas por algumas das crianças deste estudo talvez não interferissem de forma proeminente na dinâmica do cotidiano da criança e suas relações interpessoais, como há relatos semelhantes na literatura da área (Pílger \& Abreu, 2007; Silva, 2001; Silva et al., 2000). As interferências no comportamento social das crianças podem variar conforme as próprias características da doença, como a gravidade, a duração, o tratamento (Carrillo et al., 2002), sendo estes dados não especificados detalhadamente nesta pesquisa. Para entender melhor como se comportam as relações entre saúde e habilidades sociais outras pesquisas devem ser realizadas com controle de outras variáveis, como por exemplo, as limitações da doença tanto orgânica quanto social, o tempo de tratamento e até considerar problemas de comportamento enquanto grupo clínico, o que poderia gerar outras análises quando relacionado a habilidades sociais.

Em resumo, como esperado, tais resultados sugerem que o perfil do desempenho das habilidades sociais na amostra deste estudo está associado a certas características pessoais e que o padrão de relacionamento interpessoal está de acordo com o que tem sido apontado na literatura em estudos anteriores com populações semelhantes (Garaigordobil \& Maganto, 2011; Welsh et al., 2001).

No que diz respeito aos resultados de habilidades sociais nos indicadores de Frequência, aferiu-se que as crianças emitiram menos vezes (valores abaixo da média de referência) reações não-habilidosas ativas, ou seja, comportamentos considerados inadequados (comportamentos agressivos, por exemplo). Em relação ao indicador de Adequação, averiguou-se que as crianças conseguiram discernir respostas adequadas e não adequadas para os três tipos de reação, e consideraram mais certo emitir respostas habilidosas do que reações não-habilidosas passivas e não-habilidosas ativas. O desempenho do indicador
Dificuldade sugere que as crianças apesar de possuírem dificuldade de emissão das respostas socialmente habilidosas, estas foram significativamente abaixo da média. Comparando-se os resultados obtidos com a população pesquisada e os valores de referência do IMHSC-Del Prette, observou-se que o repertório de habilidades sociais permaneceu na média em relação à Frequência de emissão de reações habilidosas, sem diferenças entre as médias da amostra e a de referência.

Os dados apurados neste estudo são importantes, pois, como aponta a literatura, crianças com adequado repertório de habilidades sociais possuem menores chances de desenvolverem comportamentos antissociais ou de risco, como argumentam Arslan et al. (2011), Moura et al. (2008), Pavarino et al. (2005), Welsh et al. (2001). Considera-se que as variáveis pessoais estudadas ainda que tenham sido aqui apresentadas e discutidas de forma recortada, devem ser contextualizadas, isto é, precisam ser vistas como produto e produtora de interações do organismo vivo com o ambiente do qual faz parte. Portanto, cada variável que descreve uma característica pessoal da criança deve ter implicações para a aquisição de habilidades sociais na sua trajetória desenvolvimental. Nela, as características pessoais devem ser pensadas de maneira particular, mas também em interação com outros fatores, como as normas e valores que permeiam os grupos com os quais as crianças interagem, posto que são os processos relacionais que direcionam o curso do desenvolvimento da criança (Bronfenbrenner, 2011), e neste sentido, o domínio das habilidades sociais (Del Prette \& Del Prette, 2009).

\section{Considerações Finais}

Os resultados desta pesquisa corroboram os apontados na literatura sobre relações entre habilidades sociais e sexo e idade, porém não apresentaram associação significativa com outras variáveis pessoais como condição clínica e tratamento de saúde das crianças. Os resultados do IMHSC-Del Prette indicaram desempenho satisfatório das crianças da região periférica de Belém, na avaliação de seu repertório de reações habilidosas e não-habilidosas, o que sugere a capacidade entre elas para lidar com demandas de interação com outras crianças e adultos, sendo este resultado de relevância para a literatura da área, no quadro de avaliações de habilidades sociais de crianças brasileiras.

Diante dos resultados apresentados do desempenho das habilidades sociais e sua relação com variáveis pessoais das crianças, nota-se que a interação que ocorre entre as características investigadas e seu desempenho social pode variar conforme características particulares da pessoa. Ao mesmo tempo, estas considerações deixam claro que tais variáveis reproduzem elementos do contexto imediato em que convivem e interagem (Bronfenbrenner, 2011) e que o padrão dessa interação pode direcionar o curso do desenvolvimento do indivíduo, particularmente no que se refere às habilidades sociais. 
Em função disso, uma limitação deste estudo seja justamente o fato de as habilidades sociais terem sido avaliadas apenas a partir da versão da autoavaliação das crianças, pois apesar de ser vantajoso por acessar direto o próprio participante, pode refletir respostas socialmente aceitas e não o desempenho real que a criança teria (Del Prette \& Del Prette, 2005). Além disso, apenas uma forma de avaliação é restrita ao que o instrumento fornece, e, portanto, as situações apresentadas não contemplam as mais diversas reações e experiências pelas quais a criança continuamente passa, uma vez que alguns escolares durante a aplicação falaram espontaneamente de outras reações habilidosas e não-habilidosas que teriam diante das situações apresentadas como, por exemplo, pedir ajuda para própria professora ao invés de pedir ao colega como é apresentado no instrumento. Outra limitação apontada é a falta de um instrumento que mensure especificamente problemas de comportamento que complementariam o quadro comportamental social das crianças avaliadas e geraria outras possibilidades de análise entre as variáveis, como por exemplo, avaliar problemas de saúde apenas em grupo clínico para problemas de comportamento.

Sugere-se, assim, a utilização de outros instrumentos e técnicas como a de observação para complementar o entendimento das relações criança-criança e criança-adulto, tanto em sala de aula quanto em situação livre. Acrescenta-se que novos estudos devem ser realizados para aprofundar peso de aspectos das condições clínicas da criança como restrições na rotina que a doença impõe, e estudos longitudinais em uma amostra que permita o acompanhamento da aquisição ou redução de habilidades sociais conforme o avançar da idade e sua relação com sua condição de gênero.

\section{Referências}

Abdi, B. (2010). Gender diferences in social skills, problem behaviors and academic competence of Iranian kindergarten children based on their parent and teacher ratings. Procedia Social and Behavioral Sciences, 5, 1175-1179. doi:10.1016/j. sbspro.2010.07.256

Arslan, E., Durmusoglu-Saltali, N., \& Yilmaz, H. (2011). Social skills and emotional behavioral traits of preschool children. Social Behavior and Personality, 39(9), 1281-1288. doi:10.2224/sbp.2011.39.9.1281

Bee, H. (2003). A criança em desenvolvimento (M. A. V. Veronese, Trad.). Porto Alegre, RS: Artmed.

Berry, D., \& O'Connor, E. (2010). Behavioral risk, teacher-child relationships, and social skills development across middle childhood: A child-by-environment analysis of change. Journal of Applied Development Psychology, 31, 1-14. doi:10.1016/j.appdev.2009.05.001

Bolsoni-Silva, A. T., Del Prette, Z. A. P., Del Prette, G., Montagner, A. R., Bandeira, M., \& Del Prette, A. (2006). Habilidades sociais no Brasil: Uma análise dos estudos publicados em periódicos. In M. Bandeira, Z. A. P. Del Prette, \& A. Del Prette (Eds.), Estudos sobre habilidades sociais e relacionamento interpessoal (pp. 1-45). São Paulo, SP: Casa do Psicólogo.

Bolsoni-Silva, A. T., Marturano, E. M., \& Freiria, L. R. B. (2010). Indicativos de problemas de comportamento e de habilidades sociais em crianças: Um estudo longitudinal. Psicologia: Reflexão e Crítica, 23(3), 506-515. doi:10.1590/ S0102-79722010000300011

Boyer, B. P., \& Nelson, J. A. (2015). Longitudinal associations of childhood parenting and adolescent health: The mediating influence of social competence. Child Development, 86(3), 828-843. doi:10.1111/cdev. 12347

Bronfenbrenner, U. (2011). Bioecologia do desenvolvimento humano: Tornando os seres humanos mais humanos. Porto Alegre, RS: Artmed.

Caballo, V. E. (2012). Manual de avaliação e treinamento das habilidades sociais (S. M. Dolinsky, Trad.). São Paulo, SP: Santos.

Campanha, S. M. A., Freire, L. M. S., \& Fontes, M. J. F. (2008). $\mathrm{O}$ impacto da asma, da rinite alérgica e da respiração oral na qualidade de vida de crianças e adolescentes. Revista CEFAC, 10(4), 513-519. doi:10.1590/S1516-18462008000400011

Carrillo, A., Jiménez, V., Loubat, M., Castillo, R., Marín, F., Carvajal, M., ...Cuadra, L. (2002). Estudio descriptivo-comparativo de la calidad de vida de niños, entre 6-10 años, com epilepsia refractaria y no refractaria al tratamento. Revista Chilena de Epilepsia, 3(3), 4-10.

Cecconello, A. M., \& Koller, S. H. (2000). Competência social e empatia: Um estudo sobre resiliência com crianças em situação de pobreza. Estudos de Psicologia (Natal), 5(1), 71-93.

Del Prette, Z. A. P., \& Del Prette, A. (2005). Sistema multimídia de habilidades sociais de crianças (SMHSC-Del Prette): Manual. São Paulo, SP: Casa do Psicólogo.

Del Prette, Z. A. P., \& Del Prette, A. (2009). Psicologia das habilidades sociais na infância: Teoria e prática. Petrópolis, RJ: Vozes.

Fumo, V. M. S. (2009). Habilidades sociais acadêmicas de crianças com baixo e alto rendimento acadêmico na interação com o professor (Dissertação de mestrado, Programa de Pós-Graduação em Educação Especial, Universidade Federal de São Carlos, SP, Brasil).

Fumo, V. M. S., Manolio, C. L., Bello, S., \& Hayashi, M. C. P. I. (2009). Produção científica em habilidades sociais: Estudo bibliométrico. Revista Brasileira de Terapia Comportamental e Cognitiva, 11(2), 246-266.

Garaigordobil, M., \& Maganto, C. (2011). Empatía y resolución de conflictos durante la infancia y la adolescencia. Revista Latinoamericana de Psicologia, 43(2), 255-266.

Gon, M. C. C., Menezes, M. C., Jacovozzi, F. M., \& Zazula, R. (2013). Perfil comportamental de crianças com dermatoses crônicas de acordo com avaliação de cuidadores. Psico (Porto Alegre), 44(2), 167-173.

Lima, L., Guerra, M. P., \& Lemos, M. S. (2010). The psychological adjustment of children with asthma: Study of associated variables. The Spanish Journal of Psychological, 13(1), 353363. doi:10.1017/S1138741600003917

Moura, C. B., Marinho-Casanova, M. L., Meurer, P. H., \& Campana, C. (2008). Caracterização da clientela pré-escolar de uma clínica-escola brasileira a partir do Child Behavior Checklist (CBCL). Contextos Clínicos, 1(1), 1-8.

Ortiz, D. F., Pupo, M. C., Soto, S. G., Gutiérrez, M. J. C., Rodríguez, R. A., Marie, G. C., ...Valdés, R. R. (2005). Factores psicológicos que contribuyen al asma en niños y adolescentes asmáticos y sus padres. Revista Alergia México, 54(4), 161-170.

Pavarino, M. G., Del Prette, A., \& Del Prette, Z. A. P. (2005) O desenvolvimento da empatia como prevenção da agressividade na infância. Psico (Porto Alegre), 36(2), 127-134. 
Pichardo, M. C., García, T., Justicia, F., \& Llanos, C. (2008). Efectos de un programa de intervención para la mejora de la competencia social en niños de educación primaria en Bolivia. International Journal of Psychology and Psychological Therapy, 8(3), 441-452.

Pílger, C., \& Abreu, I. S. (2007). Diabetes mellitus na infância: Repercussões no cotidiano da criança e de sua família. Cogitare Enfermagem, 12(4), 494-501. doi:10.5380/ce.v12i4.10076

Sabol, T. J., \& Pianta, R. C. (2011). Patterns of school readiness forecast achievement and socioemotional development at the end of elementary school. Child Development, 83(1), 282-299. doi:10.1111/j.1467-8624.2011.01678.x

Salomão, J. B., Jr., Miyazaki, M. C. O. S., Cordeiro, J. A., Domingos, N. A. M., \& Valerio, N. I. (2008). Asma, competência social e transtornos comportamentais em crianças e adolescentes. Estudos de Psicologia (Campinas), 25(2), 185-192. doi:10.1590/S0103-166X2008000200003

Silva, M. G. N. (2001). Doenças crônicas na infância: Conceito, prevalência e repercussões emocionais. Revista de Pediatria do Ceará, 2(2), 29-32.

Silva, M. G. N., Naspitz, C. K., \& Solé, D. (2000). Qualidade de vida nas doenças alérgicas: Por que é importante avaliar? Revista Brasileira de Alergia e Imunopatologia. Recuperado em http://www.asbai.org.br/revistas/Vol236/qual.htm

Valle, T. G. M., \& Garnica, K. R. H. (2009). Avaliação e treinamento de habilidades sociais de crianças em idade pré-escolar. In T. G. M. Valle (Ed.), Aprendizagem e desenvolvimento humano: Avaliações e intervenções (pp. 48-75). São Paulo, SP: Cultura Acadêmica.

Vugt, E. S., Dekovic, M., Prinzie, P., Stams, G. J. J. M., \& Asscher, J. J. (2013). Evaluation of a group-based social skills training for children with problem behavior. Children and Youth Services Review, 35, 162-167. doi:10.1016/j.childyouth.2012.09.022

Welsh, M., Parke, R. D., Widaman, K., \& O’Neil, R. (2001). Linkages between children's social and academic competence: A longitudinal analysis. Journal of School Psychology, 39(6), 463-481. doi:10.1016/S0022-4405(01)00084-X

Zahn-Waxler, C., Shirtcliff, E. A., \& Marceau, K. (2008). Disorders of childhood and adolescence: Gender and psychopathology. Annual Review of Clinical Psychology, 4, 275-303. doi:10.1146/annurev.clinpsy.3.022806.091358

Zbikowski, S. M., \& Cohen, R. (1998). Parent and peer evaluations of the social competence of children with mild asthma. Journal of Applied Developmental Psychology, 19(2), 249265. doi:10.1016/S0193-3973(99)80039-2 